\title{
Queixas osteomusculares entre pescadores artesanais da cidade de Santarém - Pará
}

\author{
Osteomuscular complaints among artisan fishermen in the city of Santarém-Pará \\ Quejas osteomusculares entre pescadores artesanos en la ciudad de Santarém-Pará
}

Daliane Ferreira Marinho"* ${ }^{1 *}$ Carlos Alberto Cavalcante Gomes², Kamila Nayane Imbelloni Martins², Mayara Renata Lima Mota ${ }^{2}$, Luana Damasceno Ferreira'2, Robert Douglas Costa de Melo², Karen Evelin Pedroso de Sousa ${ }^{2}$, Eliane Ferreira Marinho ${ }^{3}$, Delma Pessanha Neves ${ }^{4}$.

\section{RESUMO}

Objetivo: Levantar o índice de queixas osteomusculares entre pescadores artesanais da cidade de SantarémPará, filiados a Colônia de Pescadores Z-20 (CP Z-20). Métodos: Tratou-se de uma pesquisa de campo, descritiva, transversal e com abordagem quantitativa. Contou com uma amostra de 111 indivíduos, ambos os sexos, idade de 18 a 70 anos. Os participantes foram questionados quanto a suas queixas osteomusculares nos últimos 12 meses e nos últimos 7 dias. Os dados foram analisados através de estatística descritiva (porcentagens, média e desvio padrão). O estudo foi aprovado por Comitê de Ética em Pesquisa com seres humanos. Resultados: A amostra foi composta por $(n=111)$ de pescadores artesanais associados à CP Z20. Destes, $73,2 \%$ eram homens e $26,8 \%$ eram mulheres. As queixas osteomusculares mais comuns relatadas por eles foram nas regiões: pescoço, parte superior das costas e parte inferior das costas, nos últimos 12 meses. Sendo esta última também a mais relatada por eles nos últimos 7 dias. Conclusão: Foi possível levantar o índice de queixas osteomusculares entre o público estudado e assim analisar o cenário atual da saúde ortopédica dessa população. Porém, a amostra poderia ter sido maior, o que se recomenda para pesquisas futuras.

Palavras-chave: Pesca, Saúde, Dor muscular.

\section{ABSTRACT}

Objective: To count the musculoskeletal complaints index among artisanal fishermen in the city of SantarémPará, affiliated to the Z-20 Fishermen's Colony (CP Z-20). Methods: This was a field research, descriptive, cross-sectional and quantitative approach. This study had a sample of 111 individuals, both sexes, aged 18 to 70 years. Participants were asked about their musculoskeletal complaints in the last 12 months and the last 7 days. Data were analyzed using descriptive statistics (percentages, mean and standard deviation). The study was approved by the Human Research Ethics Committee. Results: The sample consisted of $(n=111)$ artisanal fishermen associated with CP Z-20. Of these, $73.2 \%$ were men and $26.8 \%$ were women. The most common musculoskeletal complaints they reported were in the regions: neck, upper back, and lower back, in the last 12 months. The latter being also the most reported by them in the last 7 days. Conclusion: It was possible to count the index of musculoskeletal complaints among the studied population and thus analyze the current scenario of orthopedic health of this population. However, the sample could have been larger, which is recommended for future research.

Key words: Fishing, Health, Muscle pain.

1 Universidade Federal do Oeste do Pará (UFOPA), Santarém - PA. *E-mail: dalianemarinho@yahoo.com.br

2 Universidade do Estado do Pará (UEPA), Santarém - PA.

${ }^{3}$ Centro Universitário da Amazônia (UNAMA), Santarém - PA.

${ }^{4}$ Universidade Federal Fluminense (UFF), Rio de Janeiro - RJ. 


\section{RESUMEN}

Objetivo: Elevar el índice de quejas musculoesqueléticas entre los pescadores artesanales de la ciudad de Santarém-Pará, afiliados a la Colonia de Pescadores Z-20 (CP Z-20). Métodos: Fue un enfoque de investigación de campo, descriptivo, transversal y cuantitativo. Tenía una muestra de 111 individuos, de ambos sexos, de 18 a 70 años. Se preguntó a los participantes sobre sus quejas musculoesqueléticas en los últimos 12 meses y los últimos 7 días. Los datos se analizaron mediante estadística descriptiva (porcentajes, media y desviación estándar). El estudio fue aprobado por el Comité de Ética de Investigación Humana. Resultados: La muestra consistió en $(n=111)$ pescadores artesanales asociados con CP Z-20. De estos, el $73.2 \%$ eran hombres y el $26.8 \%$ mujeres. Las quejas musculoesqueléticas más comunes que informaron fueron en las regiones: cuello, espalda superior y espalda baja en los últimos 12 meses. Este último también es el más reportado por ellos en los últimos 7 días. Conclusión: Fue posible cuantificar el índice de quejas musculoesqueléticas entre la población estudiada y así analizar el escenario actual de salud ortopédica de esta población. Sin embargo, la muestra podría haber sido más grande, lo que se recomienda para futuras investigaciones.

Palabras clave: Pesca, Salud, Dolor muscular.

\section{INTRODUÇÃO}

Entende-se por pesca a ação, ato ou a operação desenvolvida com a finalidade de extrair, colher, apanhar, apreender ou capturar recursos pesqueiros. A pesca artesanal é uma atividade produtiva, uma modalidade de pesca comercial, que se caracteriza pelo trabalho pouco mecanizado, de forma autônoma e em regime de economia familiar, e que emprega, como meio de realização, motores de pouca potência em pequenas embarcações (BRASIL SS, 2009). De acordo com o Ministério da Pesca e Aquicultura o total de pescadores ativos e registrados no Brasil em 2010 era de 853.231 pessoas. Em se tratando da região Norte, onde foi realizado este estudo, o número de pescadores ativos em 2010 era de 330.749 pescadores. E é no norte do país que ocorre a maior produção de pescado de água doce do país, correspondendo a 55,7\% (BRASIL, 2012). Segundo Isaac VJ, et al. (2005), na cidade de Santarém, no Estado do Pará, o desembarque de pescado gira em torno de aproximadamente 4.000 toneladas ao ano. É expressiva a comercialização do produto em âmbito municipal e regional. $\mathrm{O}$ que demonstra a grande importância dessa atividade para a população local e sua economia (LIMA KF, et al., 2016).

A atividade da pesca, segundo informações da Organização Internacional do Trabalho (OIT), se caracteriza como uma atividade produtiva altamente perigosa e que oferece muitos riscos a saúde do trabalhador (VIEGAS C, 2008; OIT, 1998). Quanto aos acidentes de trabalho mais comuns entre pescadores artesanais, no Estado do Pará temos: o contato com ferrões e picadas de animais e acidentes com instrumentos de trabalho, como arpões, anzóis, redes de pesca, entre outros (NOGUEIRA LSM, et al., 2017). Sendo outro agravo comum os afogamentos, que inevitavelmente levam os trabalhadores a óbito.

Já em relação aos adoecimentos, de acordo com Viegas C (2008), os mais frequentemente encontrados entre os pescadores foram: hipertensão; doenças mentais; doenças descompressivas; doenças oculares e dermatológicas - como o câncer de pele, devido ao excesso de exposição à radiação solar. Além de doenças ocasionadas por fatores ergonômicos, como lesões osteomusculares ocasionadas por posturas inadequadas e esforços repetitivos prolongados, em membros superiores, inferiores e na coluna, como: lombalgias, hérnia de disco, e tendinites, por exemplo (PENA PGL e GOMEZ CM, 2014).

Distúrbios relacionados ao sistema musculoesquelético geram grande prejuízo para a saúde e consequentemente para a capacidade laboral do pescador. E diante dessa problemática e da ausência de dados estatísticos sobre as queixas osteomusculares entre pescadores artesanais da cidade de Santarém, no Estado do Pará, este estudo teve por objetivo levantar o índice de queixas osteomusculares entre pescadores artesanais dessa cidade, filiados a Colônia de Pescadores Z-20. A fim de traçar um perfil de tais queixas e assim poder subsidiar dados que apoiem ações preventivas futuras.

REAS/EJCH | Vol.12(3) | e2572 | DOI: https://doi.org/10.25248/reas.e2572.2020 Página 2 de 8 


\section{MÉTODOS}

O estudo foi realizado através de uma pesquisa de campo, de natureza descritiva e exploratória, do tipo transversal, com abordagem quantitativa. Teve como público-alvo pescadores artesanais associados à Colônia de pescadores Z-20 (CP Z-20), localizada na cidade de Santarém, Pará, Brasil. A amostra foi composta por $(n=111)$ pescadores artesanais, que foram selecionados de forma aleatória mediante convite verbal feito durante as reuniões mensais nos núcleos de base da região urbana. Seguindo os critérios de inclusão da pesquisa: Pescadores de ambos os sexos, com idade superior a 18 anos, associados à CP Z-20 a mais de 12 meses e em atividade, capazes de compreender e responder aos instrumentos de pesquisa, e que aceitassem participar voluntariamente da pesquisa mediante a assinatura do Termo de Consentimento Livre e Esclarecido (TCLE). E como critérios de exclusão pescadoras grávidas em qualquer fase da gestação.

A coleta de dados ocorreu no período de junho a outubro de 2019. E utilizou como instrumentos de coleta de dados o Questionário Nórdico de Sintomas Osteomusculares (QNSO), criado por Kuorinka I, et al. (1987) e traduzido e validado para o português por Pinheiro FA, et al. (2002). Ao QNSO foram acrescentadas quatro perguntas: idade e sexo do(a) pescador(a), tempo de trabalho na atividade da pesca e tipos de instrumentos de pesca utilizados. A fim de compreender o perfil da amostra estudada e como tais indicadores poderiam estar influenciando no índice de queixas ortopédicas.

Os dados obtidos foram inclusos em banco de dados através de planilhas do Microsoft Excel Office® 2010 e posteriormente foram analisados através de estatística descritiva, considerando-se como variáveis porcentagens, média e desvio padrão. A pesquisa foi aprovada pelo Comitê de Ética em Pesquisa com Seres Humanos (CEP) da Universidade do Estado do Pará, Campus XII - Santarém, sob o número de parecer 2.891.305. E mediante 0 aceite da CP Z-20.

\section{RESULTADOS}

A amostra foi composta por $(n=111)$ de pescadores artesanais associados à CP Z-20. Destes $73,2 \%$ eram homens e $26,8 \%$ eram mulheres. Entre os homens a média de idade foi de 48,1 anos $( \pm 9,5)$, e entre as mulheres a média foi de 44,8 anos $( \pm 8,7)$. Tendo o pescador do sexo masculino mais jovem 26 anos e o mais velho 70 anos, e em relação ao sexo feminino a variação foi de 18 anos para a mais jovem e de 61 anos para a mais velha. Já em relação ao tempo de atuação na pesca, a média foi de 29,8 anos $( \pm 10,6)$. E quanto aos instrumentos ou arreios de pesca mais utilizados estes deveriam relatar todos àqueles que utilizavam, e os citados foram a malhadeira $(93,8 \%)$, a tarrafa $(29,5 \%)$ e o anzol $(26,8 \%)$, respectivamente (Tabela 1).

Tabela 1 - Dados sobre os instrumentos/arreios de pesca mais utilizados pelos pescadores artesanais, filiados a CP Z-20 ( $n=111)$. Santarém-PA, 2019.

\begin{tabular}{l|l|l}
\hline Instrumento/Arreio de pesca $^{*}$ & $\mathbf{N}^{\star}$ & (\%) $^{*}$ \\
\hline Malhadeira/Rede de pesca & 105 & 93,8 \\
\hline Tarrafa & 33 & 29,5 \\
\hline Anzol & 30 & 26,8 \\
\hline Caniço & 23 & 20,5 \\
\hline Espinhel & 21 & 18,8 \\
\hline Flexa & 5 & 4,5 \\
\hline Arpão & 1 & 0,9 \\
\hline
\end{tabular}

Legenda: *Os participantes poderiam relatar mais de uma opção.

Fonte: Marinho, DF, et al., 2019. 
Em relação aos sintomas osteomusculares utilizando o instrumento QNSO, os dados estão apresentados no quadro 1, sendo que as regiões mais citadas nos últimos 12 meses foram a parte inferior das costas $(78,4 \%)$, parte superior das costas $(71,2 \%)$ e o pescoço $(57,3 \%)$, e nos últimos 7 dias foi a parte inferior das costas $(55,9 \%)$.

Quadro 1 - Queixas osteomusculares por segmentos corporais relatadas por pescadores artesanais filiados a CP Z-20, (n=111), Santarém-PA, 2019.

\begin{tabular}{|c|c|c|c|c|c|c|c|c|}
\hline QNSO (n=111) & \multicolumn{8}{|c|}{ Perguntas } \\
\hline \multirow[t]{2}{*}{ Regiões do corpo } & \multicolumn{2}{|c|}{$\begin{array}{l}\text { Nos últimos } 12 \\
\text { meses, você teve } \\
\text { problemas (como } \\
\text { dor, } \\
\text { formigamento/dor } \\
\text { mência): }\end{array}$} & \multicolumn{2}{|c|}{$\begin{array}{l}\text { Nos últimos } 12 \text { meses, } \\
\text { você foi impedido de } \\
\text { realizar atividades } \\
\text { normais (por exemplo: } \\
\text { trabalho, atividades } \\
\text { domésticas e de lazer) } \\
\text { por causa desse } \\
\text { problema em: }\end{array}$} & \multicolumn{2}{|c|}{$\begin{array}{l}\text { Nos últimos } 12 \\
\text { meses, você } \\
\text { consultou algum } \\
\text { profissional da } \\
\text { área da saúde } \\
\text { (médico, } \\
\text { fisioterapeuta) por } \\
\text { causa dessa } \\
\text { condição em: }\end{array}$} & \multicolumn{2}{|c|}{$\begin{array}{l}\text { Nos últimos } 7 \\
\text { dias, você teve } \\
\text { algum problema } \\
\text { em? }\end{array}$} \\
\hline & SIM & NÃO & SIM & NÃO & SIM & NÃO & SIM & NÃO \\
\hline Pescoço & $57,3 \%$ & $42,7 \%$ & $29,7 \%$ & $70,3 \%$ & $19,8 \%$ & $80,2 \%$ & $37,8 \%$ & $62,2 \%$ \\
\hline Ombros & $42,7 \%$ & $57,3 \%$ & $20,7 \%$ & $79,3 \%$ & $18,0 \%$ & $82,0 \%$ & $27,9 \%$ & $72,1 \%$ \\
\hline $\begin{array}{l}\text { Parte superior } \\
\text { das costas }\end{array}$ & $71,2 \%$ & $28,8 \%$ & $28,0 \%$ & $72,0 \%$ & $22,5 \%$ & $77,5 \%$ & $47,8 \%$ & $52,2 \%$ \\
\hline Cotovelos & $26,1 \%$ & $73,9 \%$ & $12,6 \%$ & $87,4 \%$ & $10,8 \%$ & $89,2 \%$ & $18,9 \%$ & $81,1 \%$ \\
\hline Punhos/mãos & $47,8 \%$ & $52,2 \%$ & $16,2 \%$ & $83,8 \%$ & $14,4 \%$ & $85,6 \%$ & $26,2 \%$ & $73,8 \%$ \\
\hline $\begin{array}{l}\text { Parte inferior das } \\
\text { costas }\end{array}$ & $78,4 \%$ & $21,6 \%$ & $41,5 \%$ & $58,5 \%$ & $28,8 \%$ & $71,2 \%$ & $55,9 \%$ & $44,1 \%$ \\
\hline Quadril/coxas & $47,8 \%$ & $52,3 \%$ & $20,7 \%$ & $79,3 \%$ & $16,2 \%$ & $83,8 \%$ & $33,3 \%$ & $66,7 \%$ \\
\hline Joelhos & $44,1 \%$ & $55,9 \%$ & $25,3 \%$ & $74,7 \%$ & $12,6 \%$ & $87,4 \%$ & $25,2 \%$ & $74,8 \%$ \\
\hline Tornozelos/pés & $31,5 \%$ & $68,5 \%$ & $13,5 \%$ & $86,5 \%$ & $9,9 \%$ & $90,1 \%$ & $20,7 \%$ & $79,3 \%$ \\
\hline
\end{tabular}

Observação: Marcados em negrito os dados mais expressivos quanto a ocorrência ou não de queixas osteomusculares/segmentos corporais/perguntas.

Fonte: Marinho, DF, et al., 2019.

\section{DISCUSSÃO}

As disfunções osteomusculares, caracterizadas principalmente por dores em uma ou mais regiões do corpo, são as principais queixas ortopédicas entre pescadores artesanais. Com o objetivo de verificar tal situação foi realizado esse estudo com 111 pescadores artesanais da cidade de Santarém-Pará. Os resultados mostraram que a relação entre pescadores do gênero masculino $(73,2 \%)$ e feminino $(26,8 \%)$ encontradas nessa pesquisa seguem a mesma tendência observada em outras pesquisas já realizadas com esse público. E também os índices governamentais conhecidos. Onde sempre há uma superioridade na proporção de homens como pescadores artesanais em relação às mulheres (ALENCAR CAG e MAIA LP, 
2011; CRUZ RC, et al., 2009; FRAGOSO JR, et al., 2018). Quanto ao perfil de idade dos pescadores artesanais abordados nessa pesquisa, as médias de idade encontradas foram de 48,1 anos $( \pm 9,5)$ para homens e de 44,8 anos $( \pm 8,7)$ para as mulheres. Sendo que dados semelhantes quanto à média de idade foram encontrados na pesquisa (RIBEIRO CRB, et al., 2015).

Na pesquisa de Fragoso JR, et al. (2018), em relação à análise por faixa etária, a maioria dos pescadores da amostra encontrava-se dentro da faixa etária que correspondia a um intervalo de idade de 40 a 59 anos, com um índice nessa faixa para os homens de $69,3 \%$ e para as mulheres de $73 \%$. Já nas pesquisas de Nogueira LSM, et al. (2017) e Ribeiro CRB, et al. (2015) os índices foram um pouco diferentes, com a maioria estando na faixa etária de 30 a 49 anos na primeira, e de 24 a 68 anos na segunda, para ambos os sexos. No entanto, observou-se entre as pesquisas consultadas que não existiu um padrão uniforme na estratificação das faixas de idade para a análise, o que resultou em dificuldades para realização de uma comparação precisa.

No presente estudo houve uma diferença quanto a forma de análise dos dados referentes à idade dos pescadores, pois esta foi feita com divisão por gênero, mas ainda assim, os resultados foram muito semelhantes à pesquisa de Ribeiro CRB, et al. (2015), com uma variação de 26 a 70 anos para os homens, e de 18 a 61 anos para as mulheres. O que demonstrou que o perfil etário na cidade de Santarém não diferiu do de outras regiões do país. Portanto, todos esses estudos confirmaram que, em sua grande maioria, os pescadores brasileiros, e também os de Santarém, se encontram na faixa de idade economicamente ativa, e dessa forma, qualquer tipo de adoecimento pode significar queda na sua capacidade laborativa e consequentemente na sua produtividade, sem falar na perda de sua qualidade de vida.

Quanto ao tempo de atuação profissional na atividade da pesca, os dados encontrados nesse estudo (média 29,8 anos) foram superiores à média obtida na pesquisa de Campos AG e Chaves JV (2016), com 16,4anos. Uma dedução possível, na ausência de dados quanto à idade dos pescadores na pesquisa destes autores seria que isso pode ter ocorrido devido terem utilizado informações antigas dos pescadores, de um banco de dados do ano 2013, e/ou devido ainda a separação em categorias de pescadores que estes fizeram, entre pescadores profissionais e pescadores de subsistência. O que não confere uma adequada categorização da classe.

Já na pesquisa de Fragoso JR, et al. (2018), estes abordaram o tempo de atuação na pesca por faixa de tempo, e a faixa mais citada entre a amostra estudada foi a de 11 a 20 anos de trabalho para homens (36,7\%) e 1 a 10 anos para mulheres (30\%). Tal diferença deve-se provavelmente a amostra pequena que obtiveram. Ou ainda ao fato de o tempo inferior de trabalho das mulheres na atividade ocorrer em virtude delas, geralmente, só assumirem a pesca como profissão após o casamento, e não desde a infância ou a juventude como os homens.

Em se tratando dos sintomas osteomusculares levantados pelos QNSO, entre a população estudada, os segmentos corporais com queixas osteomusculares nos últimos 12 meses mais citados foram: "pescoço" (57,3\%), "parte superior das costas" (71,2\%) e "parte inferior das costas" (78,4\%) (CHAGAS RA, et al., 2016; MORAES DSF, 2014; SALES VP, 2014; PROSENEWICZI I e LIPPI VG, 2012; FRAGOSO JR et al., 2018).

E quanto as queixas existentes nos últimos 7 dias, a mais citada foi a região da "parte inferior das costas" (55,9\%), o que não foi levantado pela maioria das outras pesquisas citadas acima, visto que estas se ativeram a descrever a ocorrência e não a temporalidade relacionada as queixas por segmentos. Porém, nos estudos de Sousa CB, et al. (2018) a região mais citada para os últimos 7 dias também foi a parte inferior das costas e no estudo de Fragoso JR, et al. (2018) tanto homens como mulheres relataram queixas na parte superior das costas, e elas ainda tiveram maiores queixas também na região inferior das costas (coluna lombar). $O$ que demonstra um padrão uniforme entre os locais do corpo que apresentam queixas de dor ou desconforto osteomusculares entre pescadores brasileiros, independente do sexo.

Quando levamos em consideração o tempo de ocorrência dos sintomas, a relação entre os últimos 12 meses e os últimos 7 dias evidenciam a cronicidade ou agudização das queixas ou sintomas. E o fato de a região inferior das costas ou região lombar, ter sido citada em ambos os momentos evidencia a grande 
sobrecarga biomecânica que o trabalho da pesca exerce sobre esse segmento corporal, estabelecendo um nexo causal entre a lombalgia e essa atividade laboral. Isso devido às posturas corporais adotadas pelo pescador e os movimentos realizados, também devido à carga elevada e o esforço exigido, sem falar na repetitividade dos movimentos. Esses achados se assemelham aos de estudos encontrados que relatam que a lombalgia destaca-se como uma das suas principais queixas desses profissionais, uma vez que a profissão dos pescadores artesanais exige esforços físicos prolongados, sendo que esse impacto atinge cada trabalhador de forma diferente devido às variações individuais e as variações no mesmo sujeito ao longo do tempo (NOGUEIRA LSM, et al., 2017; PENA PGL, et al., 2011; OLIVEIRA CM, et al., 2017).

Esse argumento é reforçado pelos dados obtidos quanto aos instrumentos de pesca mais utilizados pelo público, que consistiram: na malhadeira ou rede de pesca, utilizada por $93,8 \%$ deles e na tarrafa por $29,5 \%$, em segundo lugar. Ambos esses arreios de pesca exigem para sua utilização a execução de posturas em flexão e rotação do tronco constante, acrescidas do peso do material molhado, somado ao peso das espécies de peixes capturados e da corrente do rio. Todos esses fatores juntos aumentam ainda mais a carga a ser superada e suportada pelo pescador durante a atividade, o que leva as desordens biomecânicas e desgaste da região lombar, ocasionando dor e limitação funcional (PENA PGL, et al., 2011; SALDANHA MCW, 2010).

Além da região lombar, tanto o pescoço como a região superior das costas também sofrem grandes sobrecargas biomecânicas durante a utilização da malhadeira ou rede de pesca e da tarrafa, o que justifica o grande número de relatos de queixas osteomusculares nessas regiões. Porém, com tendência a ser mais relacionada a um quadro crônico do que agudo na população estudada. Isso possibilita o entendimento de que ações de educação em saúde e de reabilitação devem ser tomadas a fim de diagnosticar e tratar as desordens existentes que forem passíveis de reversão, e prevenir ou minimizar agravos nas já instaladas, como também sugerem outras pesquisas (TORRES DMA, 2016; PROSENEWICZI I e LIPPI VG, 2012).

No entanto, o tratamento adequado de tais desordens esbarra na dificuldade de acesso que essa população tem aos serviços de saúde pública, devido à demora em conseguir atendimento com especialistas, em conseguir realizar exames e de ter acesso à reabilitação. Isso pode ser evidenciado nas respostas a terceira pergunta do questionário QNSO, que questionou quanto à busca por atendimento especializado, e evidenciou a baixa procura no intervalo de 12 meses. Isso apesar de terem citado queixas osteomusculares em diversos segmentos corporais nesse mesmo período (FRAGOSO JR, et al., 2018; PROSENEWICZI I e LIPPI VG (2012); VASCONCELOS LC, et al., (2012); PENA PGL, et al., 2011).

Em relação aos resultados obtidos na segunda pergunta do questionário, os participantes foram categóricos ao afirmar, em sua maioria, que apesar da existência de queixas osteomusculares, como dor e desconforto em diversos segmentos corporais, estes não foram impeditivos para realização de sua atividade laboral ou de lazer. Isso se deve talvez ao fato de que os sintomas estão em fase inicial, sem alterações mais severas, e assim ainda permitem a manutenção da rotina de trabalho, ou, e mais provável, que esses trabalhadores se submetem a sacrifícios ao trabalharem com dor ou desconforto em uma ou diversas regiões do corpo, por não encontrarem alternativa de trabalho e renda, e nem assistência de saúde adequada que trate tais sintomas. Condições semelhantes foram encontradas em pesquisas realizadas com pescadores artesanais de outras regiões do país, o que evidencia a mesma fragilidade nos aspectos da relação saúdetrabalho dessa classe (FRAGOSO JR, et al., 2018; TORRES DMA, 2016; PROSENEWICZI I e LIPPI VG, 2012; PENA PGL, et al., 2011).

A desproporção entre o percentual de homens e mulheres atuando na atividade da pesca pode se dever a diferentes fatores, mas o principal deles acredita-se ser a subnotificação. Isso deve ocorrer devido à falta de "autorreconhecimento" da maioria delas enquanto pescadoras, por não entenderem que segundo a legislação brasileira a classificação de pescador artesanal é extensível a esposa/companheira, devido a ser uma atividade desenvolvida em caráter de economia familiar. E assim, estas não se cadastram juntos aos sindicatos. Outro fator limitante é a falta de formalização da união entre o casal, o que também impossibilita o registro da mulher junto aos sindicatos. Além da atividade da pesca possuir exigências físicas muito extenuantes para a sua execução, o que acaba por realizar um processo de seleção por gênero, visto que fisiologicamente os homens possuem maior força física.

REAS/EJCH | Vol.12(3) | e2572 | DOI: https://doi.org/10.25248/reas.e2572.2020 Página 6 de 8 
As limitações da pesquisa foram relacionadas ao aumento do número da amostra, diante do número muito maior de pescadores artesanais associados à CP Z-20. Sugere-se ainda a realização de pesquisas que analisassem os aspectos ergonômicos relacionados à atividade da pesca.

\section{CONCLUSÃO}

Com a realização desse estudo foi possível constatar as queixas de dor dos pescadores artesanais através de um instrumento de pesquisa reconhecido e validado. E assim, quantificá-las, a fim de proporcionar indicadores locais, antes inexistentes para a região, e que podem ser utilizados para elaboração de ações de prevenção e intervenção, por instituições que atuam junto à saúde e segurança do trabalho, bem como pela própria colônia. Quanto às queixas osteomusculares mais comuns relatadas entre os pescadores artesanais estas foram relacionadas à dor, formigamento ou dormência nas regiões do pescoço, parte superior das costas e parte inferior das costas, nos últimos 12 meses. Apesar disso, em sua maioria, estes não relataram terem sido impedidos de realizar suas atividades laborais devido a esses acometimentos nesse período, e nem terem procurado atendimento de um profissional de saúde para tratamento. Já quanto a sintomas recentes, a região da parte inferior das costas foi a mais citada pelos pescadores, quando questionados quanto aos sintomas na última semana.

\section{REFERÊNCIAS}

1. ALENCAR CAG, MAIA LP. Perfil socioeconômico dos pescadores brasileiros. Arq. Ciên. Mar, Fortaleza, 2011, 44(3): 12-19.

2. BRASIL. MINISTÉRIO DA PESCA E AQUICULTURA. Boletim estatístico da pesca e aquicultura: Brasil 2010. Brasília, DF: 2012

3. BRASIL SS. Trabalho, adoecimento e saúde: aspectos sociais da pesca artesanal no Pará. 2009. Dissertação (Mestrado) - Universidade Federal do Pará, Instituto de Filosofia e Ciências Humanas, Programa de Pós-Graduação em Ciências Sociais, Belém, 2009.

4. CAMPOS AG, CHAVES JV. Perfil laboral dos pescadores artesanais no Brasil: Insumos para o programa seguro defeso. Mercado de trabalho, 60, abr. 2016.

5. CHAGAS RA, et al. Acidentes de trabalho e doenças ocupacionais em pescadores artesanais do município de São João de Pirabas, Nordeste Paraense. Número 56, Ano XV. Jun-Ago/2016.

6. CRUZ RC, et al. Abordagem da fisioterapia na saúde coletiva em comunidade de pescadores do litoral paranaense: um projeto de aprendizagem na proposta da UFPR setor litoral. Tempus - Actas de Saúde Coletiva, vol. 3, nํ⒉ p. 42-54, Abr-jun/2009.

7. FRAGOSO JR, et al. Musculoskeletal Disorders In Countryside Fishermen Of Amazonas-Brazil. O Mundo da Saúde, São Paulo - 2018; 42(1):248-265.

8. ISAAC VJ, et al. Pesca no Baixo Amazonas - a pesca e os recursos pesqueiros na Amazônia brasileira. Manaus: IBAMA/ ProVárzea. (2005).

9. KUORINKA I, et al. Standardised Nordic questionnaires for the analysis of musculoskeletal symptoms. Appl Ergon., Sep;18(3):233-7, 1987.

10. LIMA KF, et al. A comercialização do pescado no município de Santarém, Pará. Rev. Bras. Eng. Pesca 9(2): 01-09, 2016.

11. MORAES DSF, et al. Análise dos sintomas osteomusculares utilizando o questionário nordico em trabalhadores ribeirinhos do município de Parintins - AM, Brasil. v. 1, n. 1 (2014).

12. NOGUEIRA LSM, et al. Segurança e saúde dos pescadores artesanais no Estado do Pará. Ministério do Trabalho. Fundacentro. São Paulo. 2017.

13. OIT. Enciclopedia de salud y seguridad en el trabajo. Madrid, c1998. Ministério de Trabajo y Asuntos Sociales. Vol. III. Cap.66. 3ed. Versão espanhola.

14. OLIVEIRA CM, et al. Dores e Delícias da Pesca Artesanal: Um Olhar Para a Influência do Meio Ambiente no Trabalho e na Saúde. Revista Ensino, Saúde e Ambiente - V10 (1), pp. 187-202, Abril. 2017.

15. PENA PGL, et al. Trabalho artesanal, cadências infernais e lesões por esforços repetitivos: estudo de caso em uma comunidade de mariscadeiras na Ilha de Maré, Bahia. Ciência \& Saúde Coletiva, 16(8):3383-3392, 2011.

16. PENA PGL, GOMEZ CM. Saúde dos pescadores artesanais e desafios para a Vigilância em Saúde do Trabalhador. Ciênc. saúde coletiva, Rio de Janeiro, v. 19, n. 12, p. 4689-4698, Dez. 2014. 
17. PINHEIRO FA, et al. Validação do Questionário Nórdico de Sintomas Osteomusculares como medida de morbidade. Rev. Saúde Pública 2002;36(3):307-12

18. PROSENEWICZ I, LIPPI UG. Acesso aos Serviços de Saúde, Condições de Saúde e Exposição aos Fatores de Risco: percepção dos pescadores ribeirinhos do Rio Machado de Ji-Paraná, RO. Saúde Soc., 2012.

19. RIBEIRO CRB, et al. Impacto ambiental, trabalho e saúde de pescadores artesanais: a educação popular em foco. J. res.: fundam. Care. 2015.

20. RIOS AO, et al. Doenças em Trabalhadores da Pesca. Revista Baiana de Saúde Pública, v. 35, n. 1, p. 175-188, 2011.

21. SALDANHA MCW, et al. Ergonomia e sustentabilidade na atividade jangadeira: Construção das demandas ergonômicas na praia de Ponta Negra-RN. Ação ergonômica volume 7, número 1, 2010.

22. SALES VP. Reprodutibilidade de questionário de sintomas osteomusculares em população de pescadoras artesanais/marisqueiras do município de Saubara - Bahia. Monografia (Faculdade de medicina da Bahia). Universidade Federal da Bahia, Salvador, 2014; 46 p.

23. SOUSA CB, et al. Queixas dolorosas e percepção de risco do trabalho de homens ribeirinhos de uma comunidade do Amazonas. v. 5, n. 10 (2018).

24. SOUZA NSS, SANTANA VM. Incidência Cumulativa Anual de Doenças Musculoesqueléticas Incapacitantes Relacionadas ao Trabalho em Uma Área Urbana do Brasil. Cad. Saúde Pública, Rio de Janeiro - RJ, v. 27, n. 11, p. 2124-34, 2011.

25. TORRES DMA. Adoecimento e morte dos pescadores artesanais na Bacia de Campos - RJ. Dissertação (Mestrado em Políticas Sociais). Universidade Estadual do Norte Fluminense, Programa de Pós-graduação em Políticas Sociais, Campos dos Goytacazes, 2016; 111 p.

26. VASCONCELOS LC, et al. Trabalho, meio ambiente e saúde em comunidades marisqueiras de Sergipe. VI Colóquio Internacioanal "Educação e Contemporaneidade". São Cristovão-SE (2012).

27. VIEGAS C. Reduzindo os riscos para o povo do mar. Proteção, Novo Hamburgo, v. 21, n. 198, p. 32-49, jun. 2008. 